Pacific

Journal of

Mathematics

EMBEDDING FUNCTOR FOR CLASSICAL GROUPS AND BRAUER-MANIN OBSTRUCTION

Eva BAyer-Fluckiger, Ting-Yu LeE AND RAman PARIMAla 


\title{
EMBEDDING FUNCTOR FOR CLASSICAL GROUPS AND BRAUER-MANIN OBSTRUCTION
}

\author{
Eva Bayer-Fluckiger, Ting-Yu LeE And Raman Parimala
}

\author{
In memory of Robert Steinberg
}

\begin{abstract}
Let $K$ be a global field of characteristic not 2. The embedding problem for maximal tori in a classical group $G$ can be described in terms of algebras with involution. The aim of this paper is to give an explicit description of the obstruction group to the Hasse principle in terms of ramification properties of certain commutative étale algebras, and to show that this group is isomorphic to one previously defined by the second author. This builds on our previous work as well as on results of Borovoi. In particular, we show that this explicit obstruction group can be identified with the group of Borovoi (J. Reine Angew. Math. 473 (1996), 181-194), where $X$ is the homogeneous space associated to the embedding functor defined by the second author (Comment. Math. Helv. 89 (2014), 671-717).
\end{abstract}

\section{Introduction}

Let $K$ be a field of characteristic $\neq 2$, and let $G$ be a reductive linear algebraic group defined over $K$. The paper [Lee 2014] is concerned with embeddings of maximal tori into $G$. In particular, if $K$ is a global field, then results of Borovoi [1999] are used to show that the Brauer-Manin obstruction is the only obstruction to the Hasse principle. More precisely, the paper [Lee 2014] defines a homogeneous space $X$ over $G$ having the property that the obstruction to the Hasse principle can be seen as an element of the dual of the group $\mathrm{L}(X)$, where $\mathrm{S}(X)$ is the locally trivial subgroup of the algebraic Brauer group of $X$ (see [Borovoi 1999, p. 493, p. 499]).

If $G$ is a classical group, then the above-mentioned embedding problem can be described in terms of embeddings of algebras with involution. The aim of the present paper is to give an explicit description of the obstruction group $\mathrm{L}(X)$ in terms of ramification properties of certain commutative étale algebras.

Parimala is partially supported by National Science Foundation grant DMS-1001872.

MSC2010: 20G30.

Keywords: embedding functor, classical groups, Brauer-Manin obstruction. 
We are not aware of similar descriptions in the case of exceptional groups. Note however that when $G$ is of type $G_{2}$, the group Б $(X)$ vanishes; in particular, the Hasse principle holds (see [Beli et al. 2015, Proposition 6.1]).

The paper is structured as follows. In Section 1, we recall from [Lee 2014] the definition of the oriented embedding functor, and we discuss its relationship with embedding questions of algebras with involution. In Sections $2-5$, we assume moreover that $K$ is a global field. In these sections we give the description of the obstruction group $\mathrm{B}$, and prove that $\mathrm{B} \simeq \mathrm{5}(X)$ (see Theorem 2.1). Finally, Section 6 discusses Brauer-Manin obstructions to the Hasse principle, and the relationship of the results of the present paper with those of [Bayer-Fluckiger et al. 2014].

\section{Embedding functor, algebras with involution and orientation}

1.1. The embedding functor. Let $K$ be a field of characteristic $\neq 2$, let $K_{S}$ be a separable closure of $K$, and let $\Gamma_{K}=\operatorname{Gal}\left(K_{s} / K\right)$. Let $G$ be a reductive group over $K$. Let $T$ be a torus and let $\Psi$ be a root datum attached to $T$ (see [Demazure and Grothendieck 2011, Exposé XXI, Definition 1.1.1]). For a maximal torus $T^{\prime}$ in $G$, we let $\Phi\left(G, T^{\prime}\right)$ be the root datum of $G$ with respect to $T^{\prime}$. If $\Phi\left(G, T^{\prime}\right)_{K_{s}}$ and $\Psi_{K_{s}}$ are isomorphic, then we say that $G$ and $\Psi$ have the same type.

Assume that $G$ and $\Psi$ have the same type. Let $\underline{\operatorname{Isom}}\left(\Psi, \Phi\left(G, T^{\prime}\right)\right)$ be the scheme of isomorphisms between the root data $\Psi$ and $\Phi\left(G, T^{\prime}\right)$. Define

$$
\underline{\operatorname{Isomext}}\left(\Psi, \Phi\left(G, T^{\prime}\right)\right)=\underline{\operatorname{Isom}}\left(\Psi, \Phi\left(G, T^{\prime}\right)\right) / \mathrm{W}(\Psi),
$$

where $\mathrm{W}(\Psi)$ is the Weyl group of $\Psi$. The scheme Isomext $\left(\Psi, \Phi\left(G, T^{\prime}\right)\right)$ is independent of the choice of the maximal torus $T^{\prime}$, and we denote it by $\underline{\operatorname{Isomext}}(\Psi, G)$. An orientation is by definition an element of Isomext $(\Psi, G)(K)$.

The embedding functor $E(G, \Psi)$ is defined as follows: for any $K$-algebra $C$, let $E(G, \Psi)(C)$ be the set of embeddings $f: T_{C} \rightarrow G_{C}$ such that $f$ is both a closed immersion and a group homomorphism which induces an isomorphism $f^{\Psi}: \Psi_{C} \stackrel{\sim}{\longrightarrow} \Phi\left(G_{C}, f\left(T_{C}\right)\right)$ such that $f^{\Psi}(\alpha)=\left.\alpha \circ f^{-1}\right|_{f\left(T_{C^{\prime}}\right)}$ for all the $C^{\prime}$-roots $\alpha$ in $\Psi_{C^{\prime}}$ for each $C$-algebra $C^{\prime}$ (see [Lee 2014, §2.1]). Given an orientation $v$ in Isomext $(\Psi, G)(K)$, we define the oriented embedding functor as follows (see [Lee $2014, \S 2.2])$ : for any $K$-algebra $C$, set

$$
\begin{aligned}
E(G, \Psi, v)(C)=\left\{f: T_{C} \hookrightarrow G_{C} \mid f\right. & \in E(G, \Psi)(C) \text { and } \\
& \text { the image of } \left.f^{\Psi} \text { in } \underline{\operatorname{Isomext}}(\Psi, G)(C) \text { is } v\right\} .
\end{aligned}
$$

The oriented embedding functor is a homogeneous space under the adjoint action of $G$. For each root datum $\Psi$, we can associate a simply connected root datum $\operatorname{sc}(\Psi)$ to it (see [Demazure and Grothendieck 2011, Exposé XXI, §6.5.5 (iii)]). Let $\operatorname{sc}(T)$ be the torus associated to $\operatorname{sc}(\Psi)$. 
1.2. Algebras with involution and the embedding functor. Let $L$ be a field of characteristic $\neq 2$, and let $A$ be a central simple algebra over $L$. Let $\tau$ be an involution of $A$, and let $K$ be the fixed field of $\tau$ in $L$. Recall that $\tau$ is said to be of the first kind if $K=L$ and of the second kind if $K \neq L$; in this case, $L$ is a quadratic extension of $K$. Let $\operatorname{dim}_{L}(A)=n^{2}$. Let $E$ be a commutative étale algebra of rank $n$ over $L$, and let $\sigma: E \rightarrow E$ be a $K$-linear involution such that $\sigma|L=\tau| L$. An embedding of $(E, \sigma)$ in $(A, \tau)$ is by definition an injective homomorphism $f: E \rightarrow A$ such that $\tau(f(e))=f(\sigma(e))$ for all $e \in E$.

The unitary groups $\mathrm{U}(A, \tau)$ and $\mathrm{U}(E, \sigma)$ are defined as follows. For any commutative $K$-algebra $C$, set

$$
\mathrm{U}(A, \tau)(C)=\left\{x \in A \otimes_{K} C \mid x \tau(x)=1\right\}
$$

and

$$
\mathrm{U}(E, \sigma)(C)=\left\{x \in E \otimes_{K} C \mid x \sigma(x)=1\right\} .
$$

Let $G=\mathrm{U}(A, \tau)^{\circ}$ be the connected component of $\mathrm{U}(A, \tau)$ containing the neutral element, and let $T=\mathrm{U}(E, \sigma)^{\circ}$ be the connected component of $\mathrm{U}(E, \sigma)$ containing the neutral element.

Set $F=\{e \in E \mid \sigma(e)=e\}$. If $L \neq K$, then we have $\operatorname{dim}_{K}(F)=n$ (see for instance [Prasad and Rapinchuk 2010, Proposition 2.1]). If $L=K$, then let us assume that $\operatorname{dim}_{K}(F)=[(n+1) / 2]$.

Then one can associate a root datum $\Psi$ to the torus $T$ such that $G$ is of type $\Psi$ (see [Lee 2014, §2.3.1]). Moreover, except for $A$ of degree 2 with $\tau$ orthogonal, there exists a $K$-embedding from $(E, \sigma)$ to $(A, \tau)$ if and only if there exists an orientation $v$ such that $E(G, \Psi, v)(K)$ is nonempty (see [Lee 2014, Theorem 2.15 and Proposition 2.17]).

1.3. Orientations in terms of algebras. Let $(E, \sigma)$ and $(A, \tau)$ be as above. Assume moreover that $(A, \tau)$ is orthogonal, and that the degree of $A$ is even. Let $\Delta(E)$ be the discriminant of the étale algebra $E$ (see [Knus et al. 1998, Chapter V, $\S 18$, p. 290]), and let $Z(A, \tau)$ be the center of the Clifford algebra of $(A, \tau)$ (see [Knus et al. 1998, Chapter II (8.7)]). Then an orientation can be thought of as the choice of an isomorphism $\Delta(E) \rightarrow Z(A, \tau)$. More precisely:

Proposition 1.3.1. We have an isomorphism

$$
\underline{\operatorname{Isom}}(\Delta(E), Z(A, \tau)) \simeq \underline{\operatorname{Isomext}}(\Psi, G) .
$$

Proof. Let $E_{\tau}$ be a maximal $\tau$-invariant étale subalgebra of $A$. Let $T_{\tau}=\mathrm{U}\left(E_{\tau}, \tau\right)^{\circ}$; then $T_{\tau}$ is a maximal torus of $G$. Let $\Phi\left(G, T_{\tau}\right)$ be the root datum of $G$ with respect to $T_{\tau}$. Then we have a natural map $\alpha: \underline{\operatorname{Isom}}\left((E, \sigma),\left(E_{\tau}, \tau\right)\right) \rightarrow \underline{\operatorname{Isom}}\left(\Psi, \Phi\left(G, T_{\tau}\right)\right)$. Using the identification of $\operatorname{Aut}(E, \sigma)$ and $\operatorname{Aut}(\Psi)$, we see that $\alpha$ is equivariant under the action of $\underline{\operatorname{Aut}}(E, \sigma)$. Let $\Gamma_{0}$ be the subgroup of $\underline{\operatorname{Aut}}(E, \sigma)$ corresponding 
to the Weyl group of $\Psi$ under this identification. Note that $\Gamma_{0}$ is the twisted constant scheme which consists of even permutations in $\underline{\operatorname{Aut}}(E, \sigma) \subset \underline{\operatorname{Aut}}(E)$. Indeed, by [Lee 2014, Lemma 2.1.1 (2)] the automorphisms of $(E, \sigma)$ are in bijection with those of the root datum $\Psi$. By [Bourbaki 1981, Planche IV, numéro X], these consist of even permutations. Let us consider the following commutative diagram:

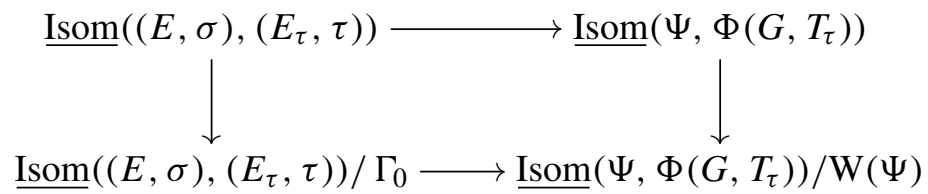

Recall that $\underline{\operatorname{Isom}}\left(\Psi, \Phi\left(G, T_{\tau}\right)\right) / \mathrm{W}(\Psi)=\underline{\operatorname{Isomext}}\left(\Psi, \Phi\left(G, T_{\tau}\right)\right)$, and note that we have $\underline{\operatorname{Isom}}\left((E, \sigma),\left(E_{\tau}, \tau\right)\right) / \Gamma_{0} \simeq \underline{\operatorname{Isom}}\left(\Delta(E), \Delta\left(E_{\tau}\right)\right)$.

If we pick another maximal étale subalgebra $E_{\tau}^{\prime}$ of $A$ invariant by $\tau$, then the method used for Isomext $\left(\Psi, \Psi_{\tau}\right)$ in [Lee 2014, §2.2.1] shows that we have a canonical isomorphism between $\underline{\operatorname{Isom}}\left(\Delta(E), \Delta\left(E_{\tau}^{\prime}\right)\right)$ and $\underline{\operatorname{Isom}}\left(\Delta(E), \Delta\left(E_{\tau}\right)\right)$.

Let us fix an isomorphism $\Delta\left(E_{\tau}\right) \rightarrow Z(A, \tau)$ as in [Bayer-Fluckiger et al. 2014, $\S 2.3]$. This gives an isomorphism $\underline{\operatorname{Isom}}\left(\Delta(E), \Delta\left(E_{\tau}\right)\right) \rightarrow \underline{\operatorname{Isom}}(\Delta(E), Z(A, \tau))$. Hence, we have

$$
\underline{\operatorname{Isom}}(\Delta(E), Z(A, \tau)) \simeq \underline{\operatorname{Isomext}}\left(\Psi, \Phi\left(G, T_{\tau}\right)\right)=\underline{\operatorname{Isomext}}(\Psi, G) .
$$

See [Bayer-Fluckiger et al. 2014, §2] for details concerning the construction and properties of orientation in terms of algebras with involution.

\section{Obstruction groups}

Assume now that $K$ is a global field, let $(E, \sigma),(A, \tau)$ be as in Section 1 , and suppose that $\tau$ is either orthogonal or unitary. Note that $L=K$ in the first case, and $L \neq K$ in the second case. The aim of this section and the following ones is to recall the definition of the obstruction group to the Hasse principle defined in [BayerFluckiger et al. 2014, §3, §5.1], and show that it is isomorphic to the obstruction group of [Lee 2014] (see Proposition 2.2), as well as to the one considered by Borovoi [1996; 1999] (see Theorem 2.1). As we will see, the group Б $(E, \sigma)$ is defined in terms of ramification properties of the algebra $(E, \sigma)$.

Let us denote by $\Omega_{K}$ the set of places of $K$. For all $v \in \Omega_{K}$, we denote by $K_{v}$ the completion of $K$ at $v$. For all $K$-algebras $B$, set $B^{v}=B \otimes_{K} K_{v}$.

The commutative étale algebra $E$ is by definition a product of separable field extensions of $L$. Let us write $E=E_{1} \times \cdots \times E_{m}$, with $\sigma\left(E_{i}\right)=E_{i}$ for all $i=1, \ldots, m$, and such that $E_{i}$ is either a field stable by $\sigma$ or a product of two fields exchanged by $\sigma$. Recall that $F=E^{\sigma}$. 
Set $I=\{1, \ldots, m\}$. We have $F=F_{1} \times \cdots \times F_{m}$, where $F_{i}$ is the fixed field of $\sigma$ in $E_{i}$ for all $i \in I$. Note that either $E_{i}=F_{i}=K$ or $E_{i}=F_{i} \times F_{i}$ or $E_{i}$ is a quadratic field extension of $F_{i}$.

Let us write

$$
E=E_{1} \times \cdots \times E_{m_{1}} \times E_{m_{1}+1} \times \cdots \times E_{m},
$$

where $E_{i} / F_{i}$ is a quadratic extension for all $i=1, \ldots, m_{1}$ and where $E_{i}=F_{i} \times F_{i}$ or $E_{i}=K$ if $i=m_{1}+1, \ldots, m$. Set $E^{\prime}=E_{1} \times \cdots \times E_{m_{1}}$ and $I^{\prime}=\left\{1, \ldots, m_{1}\right\}$. If $i \in I^{\prime}$, let $\Sigma_{i}$ be the set of places $v \in \Omega_{K}$ such that all the places of $F_{i}$ over $v$ split in $E_{i}$. Given an $m_{1}$-tuple $x=\left(x_{1}, \ldots, x_{m_{1}}\right) \in(\mathbb{Z} / 2 \mathbb{Z})^{m_{1}}$, set

$$
I_{0}=I_{0}(x)=\left\{i \mid x_{i}=0\right\}, \quad I_{1}=I_{1}(x)=\left\{i \mid x_{i}=1\right\} .
$$

Note that $\left(I_{0}, I_{1}\right)$ is a partition of $I^{\prime}$. Let $S^{\prime}$ be the set

$$
S^{\prime}=\left\{\left(x_{1}, \ldots, x_{m}\right) \in(\mathbb{Z} / 2 \mathbb{Z})^{m_{1}} \mid\left(\bigcap_{i \in I_{0}} \Sigma_{i}\right) \cup\left(\bigcap_{j \in I_{1}} \Sigma_{j}\right)=\Omega_{K}\right\},
$$

and set

$$
S=S^{\prime} \cup(0, \ldots, 0) \cup(1, \ldots, 1) .
$$

We define an equivalence relation on $S$ by

$$
\begin{aligned}
\left(x_{1}, \ldots, x_{m_{1}}\right) \sim\left(x_{1}^{\prime}, \ldots, x_{m_{1}}^{\prime}\right) \quad \text { if }\left(x_{1}, \ldots, x_{m_{1}}\right)+\left(x_{1}^{\prime}, \ldots, x_{m_{1}}^{\prime}\right)=(1, \ldots, 1) \\
\\
\text { or }\left(x_{1}, \ldots, x_{m_{1}}\right)=\left(x_{1}^{\prime}, \ldots, x_{m_{1}}^{\prime}\right) .
\end{aligned}
$$

Let us denote by $\mathrm{Б}(E, \sigma)$ the set of equivalence classes of $S$ under the above equivalence relation. It is easy to check that $\mathrm{B}(E, \sigma)$ is a group under componentwise addition (see [Bayer-Fluckiger et al. 2014, Lemma 3.1.1]). Note that in [Bayer-Fluckiger et al. 2014], the group $\mathrm{Б}(E, \sigma)$ is denoted by $\amalg\left(E^{\prime}, \sigma\right)$ (see [Bayer-Fluckiger et al. 2014, §3, §5.1]).

Set $X=E(G, \Psi, u)$. Recall that we are assuming that $\tau$ is either orthogonal (and $L=K$ ) or unitary (and $L \neq K$ ). The group $\mathrm{Б}(X)$ is defined in [Borovoi 1999, §3].

Theorem 2.1. The groups $\mathrm{Б}(E, \sigma)$ and $\mathrm{B}(X)$ are isomorphic.

This theorem is a consequence of Propositions 2.2 and 2.3 below.

Proposition 2.2. The groups $\amalg^{1}(K, \operatorname{sc}(\hat{T}))$ and $\mathrm{E}(E, \sigma)$ are isomorphic.

Proposition 2.3. The groups $\amalg^{1}(K, \operatorname{sc}(\hat{T}))$ and $\mathrm{E}(X)$ are isomorphic.

The proofs of these propositions will be given in the next sections. Let us start by introducing some notation that will be used in both proofs. For any finite separable field extension $\mathbb{N} / \mathbb{N}^{\prime}$ and any discrete $\Gamma_{\mathbb{N}}$-module $M$, set $\mathrm{I}_{\mathbb{N} / \mathbb{N}^{\prime}}(M)=\operatorname{Ind}_{\Gamma_{\mathbb{N}}}^{\Gamma_{\mathbb{N}^{\prime}}}(M)$. Note that $I_{\mathbb{N} / \mathbb{N}^{\prime}}(\mathbb{Z})$ is the character group of $R_{\mathbb{N} / \mathbb{N}^{\prime}}\left(\mathbb{G}_{m}\right)$. Let $\hat{S}_{\mathbb{N} / \mathbb{N}^{\prime}}$ be the character group of the norm-one torus $\mathrm{R}_{\mathbb{N} / \mathbb{N}^{\prime}}^{(1)}\left(\mathbb{G}_{m}\right)$. 


\section{Proof of Proposition 2.2 when $L=K$ and $\tau$ is orthogonal}

We keep the notation of the previous sections, and assume that $L=K$ and that $\tau$ is orthogonal. The aim of this section is to prove Proposition 2.2 in this case. The proof of Proposition 2.2 when $L \neq K$ is the subject matter of Section 4.

Let us consider the diagram

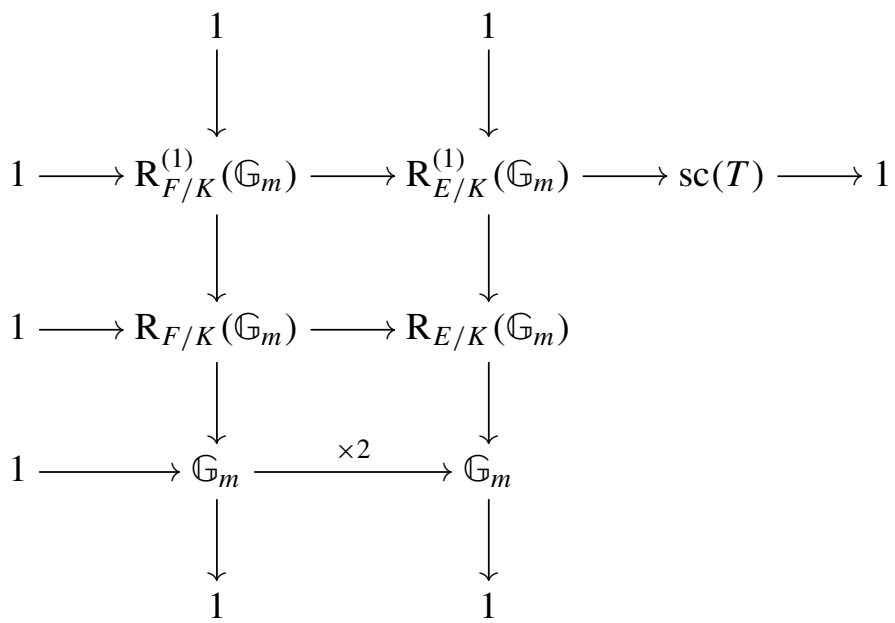

where the first row (see [Lee 2014, Lemma 3.16]) and the columns are exact. Then consider the corresponding diagram of character groups:

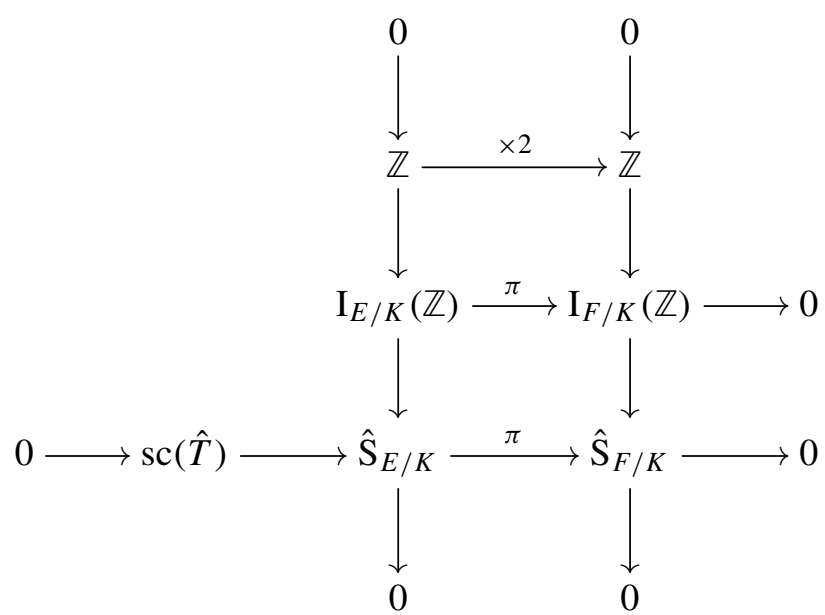

Note that we have $\mathrm{I}_{E / K}(\mathbb{Z})=\bigoplus_{i=1}^{m} \mathrm{I}_{E_{i} / K}(\mathbb{Z})$ and $\mathrm{I}_{F / K}(\mathbb{Z})=\bigoplus_{i=1}^{m} \mathrm{I}_{F_{i} / K}(\mathbb{Z})$. The module $\mathrm{I}_{E_{i} / K}(\mathbb{Z})$ can also be written as $\mathrm{I}_{F_{i} / K}\left(\mathrm{I}_{E_{i} / F_{i}}(\mathbb{Z})\right)$. Let $d$ be the degree map from $\mathrm{I}_{E_{i} / F_{i}}(\mathbb{Z}) \simeq \mathbb{Z} \oplus \mathbb{Z}$ to $\mathbb{Z}$, which sends $(x, y)$ to $x+y$. Then on each $\mathrm{I}_{F_{i} / K}\left(\mathrm{I}_{E_{i} / F_{i}}(\mathbb{Z})\right)$, the map $\pi$ is the map induced by the degree map from $\mathrm{I}_{E_{i} / F_{i}}(\mathbb{Z})$ to $\mathbb{Z}$. 
Set $\Gamma=\Gamma_{K}$. We derive the following long exact sequence from diagram (2):

$$
0 \rightarrow \operatorname{sc}(\hat{T})^{\Gamma} \rightarrow\left(\hat{\mathrm{S}}_{E / K}\right)^{\Gamma} \stackrel{\pi}{\longrightarrow}\left(\hat{\mathrm{S}}_{F / K}\right)^{\Gamma} \rightarrow \mathrm{H}^{1}(K, \mathrm{sc}(\hat{T})) \rightarrow \mathrm{H}^{1}\left(K, \hat{\mathrm{S}}_{E / K}\right) .
$$

Thus we have the exact sequence

$$
0 \rightarrow\left(\hat{\mathrm{S}}_{F / K}\right)^{\Gamma} / \pi\left(\left(\hat{\mathrm{S}}_{E / K}\right)^{\Gamma}\right) \stackrel{\delta}{\longrightarrow} \mathrm{H}^{1}(K, \operatorname{sc}(\hat{T})) \rightarrow \mathrm{H}^{1}\left(K, \hat{\mathrm{S}}_{E / K}\right) .
$$

Note that $\mathrm{H}^{2}\left(K, \mathrm{R}_{E / K}^{(1)}\left(\mathbb{G}_{m}\right)\right)$ injects into $\mathrm{H}^{2}\left(K, \mathrm{R}_{E / K}\left(\mathbb{G}_{m}\right)\right)$ by Hilbert's Theorem 90. By the Brauer-Hasse-Noether Theorem, $\amalg^{2}\left(K, \mathrm{R}_{E / K}\left(\mathbb{G}_{m}\right)\right)$ vanishes, hence so does $\amalg^{2}\left(K, \mathrm{R}_{E / K}^{(1)}\left(\mathbb{G}_{m}\right)\right)$. By Poitou-Tate duality, we have

$$
\amalg^{1}\left(K, \hat{\mathrm{S}}_{E / K}\right) \simeq \amalg^{2}\left(K, \mathrm{R}_{E / K}^{(1)}\left(\mathbb{G}_{m}\right)\right)^{*}=0 .
$$

Therefore, $\amalg^{1}(K, \operatorname{sc}(\hat{T}))$ is in the image of $\left(\hat{\mathrm{S}}_{F / K}\right)^{\Gamma} / \pi\left(\left(\hat{\mathrm{S}}_{E / K}\right)^{\Gamma}\right)$.

Since the $F_{i}$ s are field extensions of $K$, we have $\mathrm{I}_{F_{i} / K}(\mathbb{Z})^{\Gamma} \simeq \mathbb{Z}$. Thus, we have $\mathrm{I}_{F / K}(\mathbb{Z})^{\Gamma} \simeq \bigoplus_{i}^{m} \mathrm{I}_{F_{i} / K}(\mathbb{Z})^{\Gamma} \simeq \mathbb{Z}^{m}$, and $\left(\hat{\mathrm{S}}_{F / K}\right)^{\Gamma} \simeq \mathbb{Z}^{m} /(1, \ldots, 1)$.

If $E_{i}=F_{i} \times F_{i}$, then $\pi$ sends $\mathrm{I}_{E_{i} / K}(\mathbb{Z})^{\Gamma} \simeq \mathrm{I}_{F_{i} / K}(\mathbb{Z})^{\Gamma} \times \mathrm{I}_{F_{i} / K}(\mathbb{Z})^{\Gamma}$ surjectively onto $\mathrm{I}_{F_{i} / K}(\mathbb{Z})^{\Gamma} \simeq \mathbb{Z}$. If $E_{i}=K$, then $\mathrm{I}_{E_{i} / K}(\mathbb{Z}) \simeq \mathbb{Z} \simeq \mathrm{I}_{F_{i} / K}(\mathbb{Z})$. If $E_{i}$ is a quadratic field extension of $F_{i}$, the map $\pi$ sends $\mathrm{I}_{E_{i} / K}(\mathbb{Z})^{\Gamma} \simeq \mathbb{Z}$ to $\mathrm{I}_{F_{i} / K}(\mathbb{Z})^{\Gamma} \simeq \mathbb{Z}$ by multiplication by 2 . Recall that $m=m_{1}+m_{2}$, where $m_{1}$ is the number of indices $i$ such that $E_{i}$ is a quadratic field extension of $F_{i}$, and $m_{2}$ is the number of indices $i$ such that either $E_{i}=F_{i} \times F_{i}$ or $E_{i}=K$. Then we have

$$
\left(\hat{\mathrm{S}}_{F / K}\right)^{\Gamma} / \pi\left(\left(\hat{\mathrm{S}}_{E / K}\right)^{\Gamma}\right) \simeq(\mathbb{Z} / 2 \mathbb{Z})^{m_{1}} /(1, \ldots, 1) .
$$

We claim that the map $\delta:\left(\hat{\mathrm{S}}_{F / K}\right)^{\Gamma} / \pi\left(\left(\hat{\mathrm{S}}_{E / K}\right)^{\Gamma}\right) \rightarrow \mathrm{H}^{1}(K, \mathrm{sc}(\hat{T}))$ sends bijectively $\mathrm{Б}(E, \sigma)$ to $\amalg^{1}(K, \operatorname{sc}(\hat{T}))$.

Let $\left(I_{0}, I_{1}\right) \in \mathrm{Б}(E, \sigma)$, let $a$ be the corresponding element in

$$
\left(\hat{\mathrm{S}}_{F / K}\right)^{\Gamma} / \pi\left(\left(\hat{\mathrm{S}}_{E / K}\right)^{\Gamma}\right)
$$

and let $x$ be the image of $a$ in $\mathrm{H}^{1}(K, \operatorname{sc}(\hat{T}))$. We claim that $x$ is in $\amalg^{1}(K, \operatorname{sc}(\hat{T}))$. It suffices to prove that, for any $v \in \Omega_{K}$, we have $a^{v}=0$.

For a place $v \in \bigcap_{i \in \mathrm{I}_{1}} \Sigma_{i}$, we have that $E_{i}^{v}$ splits over $F_{i}^{v}$ for all $i \in \mathrm{I}_{1}$. Hence, $\pi$ maps $\mathrm{I}_{E_{i}^{v} / K_{v}}(\mathbb{Z})^{\Gamma_{v}} \simeq \mathrm{I}_{F_{i}^{v} / K_{v}}(\mathbb{Z})^{\Gamma_{v}} \oplus \mathrm{I}_{F_{i}^{v} / K_{v}}(\mathbb{Z})^{\Gamma_{v}}$ onto $\mathrm{I}_{F_{i}^{v}} / K_{v}(\mathbb{Z})^{\Gamma_{v}}$ for each $i \in \mathrm{I}_{1}$, and so $\left(\hat{\mathrm{S}}_{F / K}\right)^{\Gamma_{v}} / \pi\left(\left(\hat{\mathrm{S}}_{E / K}\right)^{\Gamma_{v}}\right)=0$ for each $i \in \mathrm{I}_{1}$ and $a_{i}^{v}=0$. On the other hand, for each $i \in \mathrm{I}_{0}$, we have $a_{i}=0$ by definition. Therefore, $a^{v}=0$.

For a place $v \in \bigcap_{i \in \mathrm{I}_{0}} \Sigma_{i}$, we replace $\left(a_{1}, \ldots, a_{m_{1}}\right)$ by $\left(a_{1}, \ldots, a_{m_{1}}\right)+(1, \ldots, 1)$. Note that $\left(a_{1}, \ldots, a_{m_{1}}\right)+(1, \ldots, 1)$ and $\left(a_{1}, \ldots, a_{m_{1}}\right)$ represent the same class $a$ in $\left(\hat{\mathrm{S}}_{F / K}\right)^{\Gamma} / \pi\left(\left(\hat{\mathrm{S}}_{E / K}\right)^{\Gamma}\right)$. By the same argument as above, we have $a_{v}=0$. Since $\left(\bigcap_{i \in \mathrm{I}_{0}} \Sigma_{i}\right) \cup\left(\bigcap_{j \in \mathrm{I}_{1}} \Sigma_{j}\right)=\Omega_{K}$, we have $a^{v}=0$ for all $v \in \Omega_{K}$, which proves that $x$ is an element of $\amalg^{1}(K, \operatorname{sc}(\hat{T}))$. 
This proves that $\delta$ induces a map $\mathrm{Б}(E, \sigma) \rightarrow \amalg^{1}(K, \operatorname{sc}(\hat{T}))$. We already know that this map is injective. Let us prove that it is also surjective.

Let $0 \neq x \in \amalg^{1}(K, \operatorname{sc}(\hat{T}))$. Let $a \in\left(\hat{\mathrm{S}}_{F / K}\right)^{\Gamma} / \pi\left(\left(\hat{\mathrm{S}}_{E / K}\right)^{\Gamma}\right)$ be the preimage of $x$, let $a^{v}$ be the localization of $a$ at the place $v$, and let $\left(a_{1}, \ldots, a_{m_{1}}\right)$ be a lift of $a$ in $(\mathbb{Z} / 2 \mathbb{Z})^{m_{1}}$. Let $\left(I_{0}, I_{1}\right)$ be the corresponding partition. Now we claim that $\left(\bigcap_{i \in \mathrm{I}_{0}} \Sigma_{i}\right) \cup\left(\bigcap_{j \in \mathrm{I}_{1}} \Sigma_{j}\right)=\Omega_{K}$. Suppose that $\left(\bigcap_{i \in \mathrm{I}_{0}} \Sigma_{i}\right) \cup\left(\bigcap_{j \in \mathrm{I}_{1}} \Sigma_{j}\right) \neq \Omega_{K}$, and let $v \in \Omega_{K} \backslash\left(\bigcap_{i \in \mathrm{I}_{0}} \Sigma_{i}\right) \cup\left(\bigcap_{j \in \mathrm{I}_{1}} \Sigma_{j}\right)$. Therefore, there exist $i_{0} \in \mathrm{I}_{0}$ and $i_{1} \in \mathrm{I}_{1}$ such that $E_{i_{0}}^{v}$ is not split over $F_{i_{0}}^{v}$ and $E_{i_{1}}^{v}$ is not split over $F_{i_{1}}^{v}$. Let $F_{i}^{v}=\prod_{j=1}^{n_{i}} L_{i, j}$, where the $L_{i, j}$ s are field extensions of $K_{v}$. Let $E_{i}^{v}=\prod_{j=1}^{n_{i}} M_{i, j}$, where $M_{i, j}$ is a quadratic étale algebra over $L_{i, j}$. Set $\Gamma_{v}=\Gamma_{K_{v}}$. Then we have

$$
\mathrm{I}_{F_{i}^{v} / K_{v}}(\mathbb{Z})^{\Gamma_{v}} / \pi\left(\mathrm{I}_{E_{i}^{v} / K_{v}}(\mathbb{Z})^{\Gamma_{v}}\right)=\bigoplus_{j=1}^{n_{i}} \mathrm{I}_{L_{i, j} / K_{v}}(\mathbb{Z})^{\Gamma_{v}} / \pi\left(\mathrm{I}_{M_{i, j} / K_{v}}(\mathbb{Z})^{\Gamma_{v}}\right) .
$$

If $M_{i, j}$ is split over $L_{i, j}$, then

$$
\mathrm{I}_{M_{i, j} / K_{v}}(\mathbb{Z})^{\Gamma_{v}}=\mathrm{I}_{L_{i, j} \times L_{i, j} / K_{v}}(\mathbb{Z})^{\Gamma_{v}}=\mathrm{I}_{L_{i, j} / K_{v}}(\mathbb{Z})^{\Gamma_{v}} \oplus \mathrm{I}_{L_{i, j} / K_{v}}(\mathbb{Z})^{\Gamma_{v}},
$$

so $\pi$ sends $\mathrm{I}_{M_{i, j} / k_{v}}(\mathbb{Z})^{\Gamma_{v}}$ surjectively to $\mathrm{I}_{L_{i, j} / k_{v}}(\mathbb{Z})^{\Gamma_{v}}$. On the other hand, if $M_{i, j}$ is a field extension over $L_{i, j}$, then $\pi$ maps $\mathrm{I}_{M_{i, j} / K_{v}}(\mathbb{Z})^{\Gamma_{v}} \simeq \mathbb{Z}$ to $2 \mathbb{Z} \subseteq \mathbb{Z} \simeq \mathrm{I}_{L_{i, j} / K_{v}}(\mathbb{Z})^{\Gamma_{v}}$ and we have

$$
\mathrm{I}_{L_{i, j} / K_{v}}(\mathbb{Z})^{\Gamma_{v}} / \pi\left(\mathrm{I}_{M_{i, j} / K_{v}}(\mathbb{Z})^{\Gamma_{v}}\right) \simeq \mathbb{Z} / 2 \mathbb{Z} .
$$

For $a_{i} \in \mathrm{I}_{F_{i} / K}(\mathbb{Z})^{\Gamma} / \pi\left(\mathrm{I}_{E_{i} / K}(\mathbb{Z})^{\Gamma}\right) \simeq \mathbb{Z} / 2 \mathbb{Z}$, the localization map sends $a_{i}$ diagonally into

$$
\mathrm{I}_{F_{i}^{v} / K_{v}}(\mathbb{Z})^{\Gamma_{v}} / \pi\left(\mathrm{I}_{E_{i}^{v} / K_{v}}(\mathbb{Z})^{\Gamma_{v}}\right) \simeq \bigoplus_{\substack{j \text { where } M_{i, j} \\ \text { is nonsplit }}} \mathbb{Z} / 2 \mathbb{Z} .
$$

Let $a_{i}^{v}$ be the image of $a_{i}$ in $\mathrm{I}_{F_{i}^{v} / K_{v}}(\mathbb{Z})^{\Gamma_{v}} / \pi\left(\mathrm{I}_{E_{i}^{v} / K_{v}}(\mathbb{Z})^{\Gamma_{v}}\right)$. By our choice of $v$, we have that $\mathrm{I}_{F_{i_{0}}^{v} / K_{v}}(\mathbb{Z})^{\Gamma_{v}} / \pi\left(\mathrm{I}_{E_{i_{0}}^{v}} / K_{v}(\mathbb{Z})^{\Gamma_{v}}\right)\left(\operatorname{resp} . \mathrm{I}_{F_{i_{1}}^{v} / K_{v}}(\mathbb{Z})^{\Gamma_{v}} / \mathrm{I}_{E_{i_{1}}^{v} / K_{v}}(\mathbb{Z})^{\Gamma_{v}}\right)$ is nontrivial. In particular, $a_{i_{1}}^{v}$ is nonzero as $a_{i_{1}}$ is nonzero. Note that

$$
\bigoplus_{i}\left(\hat{\mathrm{S}}_{F_{i}^{v} / K_{v}}\right)^{\Gamma_{v}} / \pi\left(\left(\hat{\mathrm{S}}_{E_{i}^{v} / K_{v}}\right)^{\Gamma_{v}}\right)=\frac{\bigoplus_{i} \mathrm{I}_{F_{i}^{v} / K_{v}}(\mathbb{Z})^{\Gamma_{v}} / \pi\left(\mathrm{I}_{E_{i}^{v} / K_{v}}(\mathbb{Z})^{\Gamma_{v}}\right)}{(\overline{1}, \ldots, \overline{1})},
$$

where $\overline{1}$ denotes the image of the diagonal element of $\mathrm{I}_{F_{i}^{v}} / K_{v}(\mathbb{Z})^{\Gamma_{v}}$ in

$$
\mathrm{I}_{F_{i}^{v} / K_{v}}(\mathbb{Z})^{\Gamma_{v}} / \pi\left(\mathrm{I}_{E_{i}^{v} / K_{v}}(\mathbb{Z})^{\Gamma_{v}}\right) .
$$

Since $a^{v}=0$, we have either $a_{i}^{v}=0 \in \mathrm{I}_{F_{i}^{v} / K_{v}}(\mathbb{Z})^{\Gamma_{v}} / \pi\left(\mathrm{I}_{E_{i}^{v}} / K_{v}(\mathbb{Z})^{\Gamma_{v}}\right)$ for all $i$, or $a_{i}^{v}=\overline{1} \in \mathrm{I}_{F_{i}^{v} / K_{v}}(\mathbb{Z})^{\Gamma_{v}} / \pi\left(\mathrm{I}_{E_{i}^{v} / K_{v}}(\mathbb{Z})^{\Gamma_{v}}\right)$ for all $i$. In particular, this implies that $a_{i_{0}}^{v}$ and $a_{i_{1}}^{v}$ are either both 0 or both 1 , which is a contradiction. Therefore, we have $\left(\bigcap_{i \in \mathrm{I}_{0}} \Sigma_{i}\right) \cup\left(\bigcap_{j \in \mathrm{I}_{1}} \Sigma_{j}\right)=\Omega_{K}$ and $\left(I_{0}, I_{1}\right) \in \mathrm{B}(E, \sigma)$. 


\section{Proof of Proposition 2.2 when $L \neq K$}

We keep the notation of the previous sections and assume that $L \neq K$. The aim of this section is to prove Proposition 2.2 in this case.

In this case, the torus $\operatorname{sc}(T)$ fits in the following exact sequence:

$$
1 \rightarrow \operatorname{sc}(T) \rightarrow \mathrm{R}_{F / K}\left(\mathrm{R}_{E / F}^{(1)}\left(\mathbb{G}_{m}\right)\right) \rightarrow \mathrm{R}_{L / K}^{(1)}\left(\mathbb{G}_{m}\right) \rightarrow 1 .
$$

We take the dual sequence of exact sequence (3):

$$
0 \rightarrow \hat{\mathrm{S}}_{L / K} \stackrel{\iota}{\longrightarrow} \mathrm{I}_{F / K}\left(\hat{\mathrm{S}}_{E / F}\right) \stackrel{p}{\longrightarrow} \mathrm{sc}(\hat{T}) \rightarrow 0,
$$

from which we derive the long exact sequence

$$
\cdots \rightarrow \mathrm{H}^{1}\left(K, \hat{\mathrm{S}}_{E / K}\right) \stackrel{\iota^{1}}{\longrightarrow} \mathrm{H}^{1}\left(K, \mathrm{I}_{F / K}\left(\hat{\mathrm{S}}_{E / F}\right)\right) \stackrel{p^{1}}{\longrightarrow} \mathrm{H}^{1}(K, \mathrm{sc}(\hat{T})) \rightarrow \mathrm{H}^{2}\left(K, \hat{\mathrm{S}}_{E / K}\right) .
$$

By Poitou-Tate duality, we have $\amalg^{2}\left(K, \hat{\mathrm{S}}_{E / K}\right) \simeq \amalg^{1}\left(K, \mathrm{R}_{E / K}^{(1)}\left(\mathbb{G}_{m}\right)\right)^{*}$. We claim that $\amalg^{2}\left(K, \hat{\mathrm{S}}_{E / K}\right) \simeq \amalg^{1}\left(K, \mathrm{R}_{E / K}^{(1)}\left(\mathbb{G}_{m}\right)\right)^{*}=0$. To see this, we consider the following exact sequence:

$$
1 \rightarrow \mathrm{R}_{L / K}^{(1)}\left(\mathbb{G}_{m}\right) \rightarrow \mathrm{R}_{L / K}\left(\mathbb{G}_{m}\right) \rightarrow \mathbb{G}_{m} \rightarrow 1
$$

By Hilbert Theorem 90, we have $\mathrm{H}^{1}\left(K, \mathrm{R}_{L / K}^{(1)}\left(\mathbb{G}_{m}\right)\right)=K^{\times} / \mathrm{N}_{L / K}\left(L^{\times}\right)$, where $\mathrm{N}_{L / K}$ is the norm map from $L$ to $K$. Since the norms of the quadratic extension $L$ over $K$ satisfy the local-global principle, we have $\amalg^{1}\left(K, \mathrm{R}_{L / K}^{(1)}\left(\mathbb{G}_{m}\right)\right)=0$. Hence $\amalg^{2}\left(K, \hat{\mathrm{S}}_{L / K}\right)=0$. Therefore, the group $\amalg^{1}(K, \operatorname{sc}(\hat{T}))$ is contained in the image of $\mathrm{H}^{1}\left(K, \mathrm{I}_{F / K}\left(\hat{\mathrm{S}}_{E / F}\right)\right)$.

Let us consider the following exact sequence:

$$
1 \rightarrow \mathbb{G}_{m} \rightarrow \mathrm{R}_{L / K}\left(\mathbb{G}_{m}\right) \stackrel{\pi}{\rightarrow} \mathrm{R}_{L / K}^{(1)}\left(\mathbb{G}_{m}\right) \rightarrow 1,
$$

where $\pi(x)=x / \sigma(x)$. Considering the dual sequence, we get

$$
0 \rightarrow \hat{\mathrm{S}}_{L / K} \rightarrow \mathrm{I}_{L / K}(\mathbb{Z}) \stackrel{d}{\longrightarrow} \mathbb{Z} \rightarrow 0,
$$

where $d$ is the degree map which maps $(a, b) \in \mathbb{Z} \oplus \mathbb{Z} \simeq \mathrm{I}_{L / K}(\mathbb{Z})$ to $a+b$. Taking the long exact sequence associated to (7), we have

$$
\mathrm{I}_{L / K}(\mathbb{Z})^{\Gamma} \stackrel{d}{\longrightarrow} \mathbb{Z} \rightarrow \mathrm{H}^{1}\left(K, \hat{\mathrm{S}}_{L / K}\right) \rightarrow \mathrm{H}^{1}\left(K, \mathrm{I}_{L / K}(\mathbb{Z})\right)=0 .
$$

Since $L$ is a quadratic field extension of $K$, we obtain

$$
\mathrm{H}^{1}\left(K, \hat{\mathrm{S}}_{L / K}\right) \simeq \mathbb{Z} / d\left(\mathrm{I}_{L / K}(\mathbb{Z})^{\Gamma}\right)=\mathbb{Z} / 2 \mathbb{Z}
$$


Similarly, we have

$$
\mathrm{H}^{1}\left(K, \mathrm{I}_{F / K}\left(\hat{\mathrm{S}}_{E / F}\right)\right)=\mathrm{H}^{1}\left(F, \hat{\mathrm{S}}_{E / F}\right)=\prod_{i=1}^{m} \mathrm{H}^{1}\left(F_{i}, \hat{\mathrm{S}}_{E_{i} / F_{i}}\right) .
$$

If $E_{i}=F_{i} \times F_{i}$, then $\mathrm{H}^{1}\left(F_{i}, \hat{\mathrm{S}}_{E_{i} / F_{i}}\right)=0$ since $d$ is surjective. If $E_{i}$ is a quadratic extension of $F_{i}$, then $\mathrm{H}^{1}\left(F_{i}, \hat{\mathrm{S}}_{E_{i} / F_{i}}\right)=\mathbb{Z} / 2 \mathbb{Z}$. Recall that $m=m_{1}+m_{2}$, where $m_{1}$ is the number of indices $i$ such that $E_{i}$ is a quadratic extension of $F_{i}$, and $m_{2}$ is the number of indices $i$ such that $E_{i}=F_{i} \times F_{i}$. Then $\mathrm{H}^{1}\left(K, \mathrm{I}_{F / K}\left(\hat{\mathrm{S}}_{E / F}\right)\right) \simeq(\mathbb{Z} / 2 \mathbb{Z})^{m_{1}}$.

The map $\iota^{1}: \mathrm{H}^{1}\left(K, \hat{\mathrm{S}}_{L / K}\right) \rightarrow \mathrm{H}^{1}\left(K, \mathrm{I}_{F / K}\left(\hat{\mathrm{S}}_{E / F}\right)\right)$ maps $\mathbb{Z} / 2 \mathbb{Z}$ diagonally into $(\mathbb{Z} / 2 \mathbb{Z})^{m_{1}}$. Therefore, we have

$$
\amalg^{1}(k, \operatorname{sc}(\hat{T})) \subseteq \operatorname{Im}\left(p^{1}\right) \simeq(\mathbb{Z} / 2 \mathbb{Z})^{m_{1}} /(1, \ldots, 1) .
$$

Let us show that $p^{1}$ maps $\mathrm{Б}(E, \sigma)$ bijectively to $\amalg(K, \operatorname{sc}(\hat{T}))$.

Let $\left(I_{0}, I_{1}\right)$ be in $\mathrm{E}(E, \sigma)$, and let $a$ in $\mathrm{H}^{1}\left(K, \mathrm{I}_{F / K}\left(\hat{\mathrm{S}}_{E / F}\right)\right)$ be the corresponding element. We want to show that $p^{1}(a)$ is an element of $\amalg^{1}(K, \operatorname{sc}(\hat{T}))$. Let $v \in \Omega_{K}$. If $v \in \bigcap_{j \in \mathrm{I}_{1}} \Sigma_{j}$, then $a^{v}=0$. Hence, it suffices to prove that, for $v \in \Omega_{K} \backslash \bigcap_{i \in \mathrm{I}_{1}} \Sigma_{i}$, we have $a^{v}=\iota_{v}^{1}(1)=\iota^{1}(1)_{v}$. Now, since $\left(\bigcap_{i \in \mathrm{I}_{0}} \Sigma_{i}\right) \cup\left(\bigcap_{j \in \mathrm{I}_{1}} \Sigma_{j}\right)=\Omega_{K}$, we have $v \in \bigcap_{i \in \mathrm{I}_{0}} \Sigma_{i}$. Consequently, $\mathrm{H}^{1}\left(F_{i}, \hat{\mathrm{S}}_{E_{i}^{v} / F_{i}^{v}}\right)=0$ for all $i \in \mathrm{I}_{0}$, and the projection of $\iota_{v}^{1}(1)$ to these components are trivial. For $i \in \mathrm{I}_{1}$, we have that $a_{i}$ and the $i$-th coordinate of $\iota^{1}(1)$ are both 1 , so their images in $\mathrm{H}^{1}\left(F_{i}^{v}, \hat{\mathrm{S}}_{E_{i}^{v} / F_{i}^{v}}\right)$ are equal. This proves that $a^{v}=\iota_{v}^{1}(1)$, hence $p^{1}\left(a^{v}\right)=0$.

We next show that the restriction of the map $p^{1}$ to $\mathrm{L}(E, \sigma)$ is surjective onto $\amalg^{1}(K, \operatorname{sc}(\hat{T}))$.

Let $a=\left(a_{1}, \ldots, a_{m_{1}}\right) \in(\mathbb{Z} / 2 \mathbb{Z})^{m_{1}} \simeq \mathrm{H}^{1}\left(K, \mathrm{I}_{E^{\sigma} / k}\left(\hat{\mathrm{S}}_{E / F}\right)\right)$ and let $\left(I_{0}, I_{1}\right)$ be the associated partition. If $a=0$ or $a=(1, \ldots, 1)$, then $a$ is in the image of $\iota^{1}$ and we have $p^{1}(a)=0 \in \amalg^{1}(K, \operatorname{sc}(\hat{T}))$. Hence, we may assume that $I_{0}$ and $I_{1}$ are nonempty.

We claim that $0 \neq p^{1}(a) \in \amalg^{1}(K, \operatorname{sc}(\hat{T}))$ if and only if $\mathrm{I}_{0}$ and $\mathrm{I}_{1}$ are nonempty and $\left(\bigcap_{i \in \mathrm{I}_{0}} \Sigma_{i}\right) \cup\left(\bigcap_{j \in \mathrm{I}_{1}} \Sigma_{j}\right)=\Omega_{K}$.

Suppose $0 \neq p^{1}(a) \in \amalg^{1}(K, \operatorname{sc}(\hat{T}))$. Let $v \in \Omega_{K} \backslash \bigcap_{i \in \mathrm{I}_{0}} \Sigma_{i}$. Then $L^{v} \not K^{v} \times K^{v}$ and we have $\mathrm{H}^{1}\left(L^{v}, \hat{\mathrm{S}}_{L^{v} / K_{v}}\right)=\mathbb{Z} / 2 \mathbb{Z}$. Let $a^{v}$ denote the localization of $a$ at $v$. Since $p^{1}(a) \in \amalg^{1}(K, \operatorname{sc}(\hat{T}))$, we have $a^{v}$ in the image of $\iota_{v}^{1}$, so either $a^{v}=0$ or $a^{v}=\iota_{v}^{1}(1)$. It suffices to show that $v \in \bigcap_{i \in \mathrm{I}_{1}} \Sigma_{i}$. Consider the $i$-th component of $(\mathbb{Z} / 2 \mathbb{Z})^{m_{1}}$, which corresponds to $\mathrm{H}^{1}\left(K, \mathrm{I}_{F_{i} / K}\left(\hat{\mathrm{S}}_{E_{i} / F_{i}}\right)\right)=\mathrm{H}^{1}\left(F_{i}, \hat{\mathrm{S}}_{E_{i} / F_{i}}\right)$. If $E_{i}$ splits over $F_{i}$ at a place $v \in \Omega_{K}$, then by the exact sequence (8), the map $d$ is surjective and $\mathrm{H}^{1}\left(F_{i}^{v}, \hat{\mathrm{S}}_{E_{i}^{v} / F_{i}^{v}}\right)=0$, which means that the $i$-th component vanishes at place $v$. Since $v \notin \bigcap_{i \in \mathrm{I}_{0}} \Sigma_{i}$, there exists an $i \in \mathrm{I}_{0}$ such that $E_{i}^{v}$ is not split over $F_{i}^{v}$. Let $F_{i}^{v}=\prod_{j=1}^{n_{i}} L_{i, j}$, where the $L_{i, j}$ s are field extensions of $K_{v}$. Let $E_{i}^{v}=\prod_{j=1}^{n_{i}} M_{i, j}$, 
where $M_{i, j}$ is a quadratic étale algebra over $L_{i, j}$. Then

$$
\mathrm{H}^{1}\left(F_{i}^{v}, \hat{\mathrm{S}}_{E_{i}^{v} / F_{i}^{v}}\right)=\prod_{j} \mathrm{H}^{1}\left(L_{i, j}, \hat{\mathrm{S}}_{M_{i, j} / L_{i, j}}\right) .
$$

By the choice of $i$, there is a $j$ such that $M_{i, j}$ is not split over $L_{i, j}$, and hence $\mathrm{H}^{1}\left(L_{i, j}, \hat{\mathrm{S}}_{M_{i, j} / L_{i, j}}\right) \neq 0$. Therefore, the projection of $\iota_{v}^{1}(1)$ to $\mathrm{H}^{1}\left(L_{i, j}, \hat{\mathrm{S}}_{M_{i, j} / L_{i, j}}\right)$ is 1 . On the other hand, the projection of $a^{v}$ to the same component is 0 since $i \in \mathrm{I}_{0}$. Therefore, $a^{v}=0$ which means that $\mathrm{H}^{1}\left(F_{i}^{v}, \hat{\mathrm{S}}_{E_{i}^{v} / F_{i}^{v}}\right)=0$ for all $i \in \mathrm{I}_{1}$, hence $v \in \bigcap_{i \in \mathrm{I}_{1}} \Sigma_{i}$. This proves that $a \in \mathrm{Б}(E, \sigma)$.

\section{The proofs of Proposition 2.3 and Theorem 2.1}

We keep the notation of the previous sections. As we will see, Theorem 2.1 follows from Propositions 2.2 and 2.3, which we'll now prove.

Proposition 2.3. The groups $\mathrm{W}^{1}(K, \mathrm{sc}(\hat{T}))$ and $\mathrm{\textrm {D }}(X)$ are isomorphic.

For a connected reductive group $H$, we denote by $H^{\text {tor }}$ the quotient of $H$ by its derived group. Note that $H^{\text {tor }}$ is a torus.

Proof of Proposition 2.3. Let $\operatorname{sc}(G)$ be the simply connected cover of $G$. Recall that $X=E(G, \Psi, u)$. Since $X$ is a homogeneous space under the adjoint action of $G$, we can view $X$ as a homogeneous space under $\operatorname{sc}(G)$. Let $x$ be in $X\left(K_{s}\right)$ and let $\bar{H}=\operatorname{Stab}_{\mathrm{sc}(G)_{K_{s}}}(x)$ be the stabilizer of $x$ over $K_{s}$. Then $\bar{H}$ is isomorphic to $\operatorname{sc}(T)_{K_{s}}$ (see [Lee 2014, Lemma 3.9]). Let $H^{m}$ be the $K$-form of the multiplicative quotient of $\bar{H}$ constructed in [Borovoi 1999, §§1.1-1.2, pp. 493-494] (note that the hypotheses of [Borovoi 1999, §1.1]. are satisfied: (1.1.1) holds since $\operatorname{sc}(G)$ is simply connected, and (1.1.2) is satisfied since $\left.\bar{H} \simeq \operatorname{sc}(T)_{K_{s}}\right)$. We have $H^{m} \simeq \operatorname{sc}(T)$ (see [Lee 2014, Lemma 3.9]). Let $i: H^{m} \rightarrow \operatorname{sc}(G)^{\text {tor }}$ be the morphism of algebraic groups constructed in [Borovoi 1999, $\S 1.2$, p. 494]. Let $\hat{H}^{m}\left(\operatorname{resp} . \operatorname{sc}(\hat{G})^{\text {tor }}\right)$ be the character group of $H^{m}$ (resp. $\left.\operatorname{sc}(G)^{\text {tor }}\right)$. We view the dual map of $i$ as a complex of finitely generated Galois modules $\operatorname{sc}(\hat{G})^{\text {tor }} \rightarrow \hat{H}^{m}$, where $\operatorname{sc}(\hat{G})^{\text {tor }}$ is in degree 0 and $\hat{H}^{m}$ is in degree 1 . Then we have $\mathrm{E}(X)=\amalg^{2}\left(K, \operatorname{sc}(\hat{G})^{\text {tor }} \rightarrow \hat{H}^{m}\right)$. This follows from [Borovoi and van Hamel 2012, Theorem 3] (note that the statement was already proved in [Borovoi 1999, Theorem 3.3] under the condition that $X\left(K_{v}\right) \neq \varnothing$ for all $v \in \Omega_{K}$, and that Theorem 3 of [Borovoi and van Hamel 2012] was conjectured in [Borovoi 1999, Conjecture 3.2]). Since $\operatorname{sc}(G)$ is semisimple, we have $\operatorname{sc}(G)^{\text {tor }}=1$. Therefore, we have $\amalg^{2}\left(K, \operatorname{sc}(\hat{G})^{\text {tor }} \rightarrow \hat{H}^{m}\right)=\amalg^{2}\left(K, 1 \rightarrow \hat{H}^{m}\right)$. On the other hand, we have $\amalg^{2}\left(K, 1 \rightarrow \hat{H}^{m}\right)=\amalg^{1}\left(K, \hat{H}^{m}\right)$ by the definition of hypercohomology. Recall that $H^{m} \simeq \operatorname{sc}(T)$. Therefore, $\mathrm{Б}(X) \simeq \amalg^{1}(K, \operatorname{sc}(\hat{T}))$.

Theorem 2.1. The groups $\mathrm{L}(E, \sigma)$ and $\mathrm{S}(X)$ are isomorphic.

Proof of Theorem 2.1. By Proposition 2.3 we have $\mathrm{Б}(X) \simeq \amalg^{1}(K, \operatorname{sc}(\hat{T}))$, and Proposition 2.2 implies that $\amalg^{1}(K, \operatorname{sc}(\hat{T})) \simeq \mathrm{Б}(E, \sigma)$. 


\section{Hasse principle and Brauer-Manin obstruction}

We keep the notation of the previous sections and assume that $K$ is a global field. In particular, $(E, \sigma)$ is an étale algebra with involution and $(A, \tau)$ is a central simple algebra with involution, as in Section 2.

The embeddings of $(E, \sigma)$ into $(A, \tau)$ were investigated in several papers; see for instance [Prasad and Rapinchuk 2010; Lee 2014; Bayer-Fluckiger et al. 2014]. In particular, Prasad and Rapinchuk proved in [2010, Theorem 5.1] that the Hasse principle holds if $\tau$ is symplectic, and they obtained partial results for $\tau$ orthogonal and unitary as well (see the introduction of the same paper).

Since the case where $\tau$ is symplectic is covered by the results of Prasad and Rapinchuk, we henceforth assume that $\tau$ is either orthogonal or unitary.

In [Bayer-Fluckiger et al. 2014] we defined the obstruction group $\mathrm{Б}(E, \sigma)$ (see Section 4 of the present paper; note that this group is denoted by $\amalg\left(E^{\prime}, \sigma\right)$ in [BayerFluckiger et al. 2014, §3, §5.1]). Under the hypothesis that $(E, \sigma)$ can be embedded into $(A, \tau)$ everywhere locally, we also defined an element $\bar{f}=\bar{f}((E, \sigma),(A, \tau))$ of $\mathrm{\textrm {B }}(E, \sigma)^{*}$ which gives a complete obstruction to the Hasse principle:

Theorem 6.1. $(E, \sigma)$ can be embedded into $(A, \tau)$ if and only if

$$
\bar{f}((E, \sigma),(A, \tau))=0 .
$$

This is proved in [Bayer-Fluckiger et al. 2014, Theorem 4.6.1 and Proposition 5.1.1].

On the other hand, Borovoi [1996] studied the Hasse principle for homogeneous spaces of connected linear algebraic groups with connected or abelian stabilizers. If $Y$ is such a space, he defined a group $\mathrm{Б}(Y)$ and, provided $Y\left(K_{v}\right) \neq \varnothing$ for all $v \in \Omega_{K}$, an element $m_{H}(Y) \in \mathrm{Б}(Y)^{*}$ such that $Y(K) \neq \varnothing$ if and only if $m_{H}(Y)=0$.

Borovoi's results were applied to the embedding problem of algebras with involution in [Lee 2014]. Recall that $G=\mathrm{U}(A, \tau)^{\circ}$ and $T=\mathrm{U}(E, \sigma)^{\circ}$ (see Section 1), and that $X=E(G, \Psi, u)$ (see Sections 1 and 4). By Theorem 2.1 we have $\mathrm{B}(E, \sigma) \simeq \mathrm{\textrm {B }}(X)$.

We don't know whether the isomorphism between $\mathrm{Б}(E, \sigma)$ and $\mathrm{\textrm {C }}(X)$ carries $\bar{f}((E, \sigma),(A, \tau))$ to $m_{H}(X)$. However, these elements vanish simultaneously, and they both provide complete obstructions to the Hasse principle. More precisely:

Theorem 6.2. Assume that $(E, \sigma)$ can be embedded into $(A, \tau)$ everywhere locally (or, equivalently, that $X\left(K_{v}\right) \neq \varnothing$ for all $\left.v \in \Omega_{K}\right)$. Then the following assertions are equivalent:

(i) $(E, \sigma)$ can be embedded into $(A, \tau)$.

(ii) $X(K) \neq \varnothing$.

(iii) $\bar{f}((E, \sigma),(A, \tau))=0$. 
(iv) $m_{H}(X)=0$.

Proof. The equivalence of (i) and (ii) follows from [Lee 2014, Theorem 2.1.5]. The equivalence of (i) and (iii) is proved in [Bayer-Fluckiger et al. 2014, Theorem 4.6.1 and Proposition 5.1.1]. Finally, the equivalence between (ii) and (iv) follows from [Borovoi 1996, Theorem 2.2].

\section{References}

[Bayer-Fluckiger et al. 2014] E. Bayer-Fluckiger, T.-Y. Lee, and R. Parimala, "Embeddings of maximal tori in classical groups and explicit Brauer-Manin obstruction", preprint, École Polytechnique Fédérale de Lausanne, Emory University, 2014.

[Beli et al. 2015] C. Beli, P. Gille, and T.-Y. Lee, "On maximal tori of algebraic groups of type $G_{2}$ ", 2015. To appear in Pacific J. Math. arXiv 1411.6808

[Borovoi 1996] M. Borovoi, "The Brauer-Manin obstructions for homogeneous spaces with connected or abelian stabilizer”, J. Reine Angew. 473 (1996), 181-194. MR 97g:11042 Zbl 0844.14020

[Borovoi 1999] M. Borovoi, "A cohomological obstruction to the Hasse principle for homogeneous spaces”, Math. Ann. 314:3 (1999), 491-504. MR 2000i:11102 Zbl 0966.14017

[Borovoi and van Hamel 2012] M. Borovoi and J. van Hamel, "Extended equivariant Picard complexes and homogeneous spaces", Transform. Groups 17:1 (2012), 51-86. MR 2891211 Zbl 1252.14030

[Bourbaki 1981] N. Bourbaki, Éléments de mathématique, Masson, Paris, 1981. Groupes et algèbres de Lie. Chapitres 4, 5 et 6. MR 83g:17001 Zbl 0483.22001

[Demazure and Grothendieck 2011] M. Demazure and A. Grothendieck, Schémas en groupes (SGA 3), Tome III: Structure des schémas en groupes réductifs (Séminaire de Géométrie Algébrique du Bois Marie 1962-64), 2nd ed., edited by P. Gille and P. Polo, Documents Mathématiques 8, Société Mathématique de France, Paris, 2011. MR 2867622 Zbl 1241.14003

[Knus et al. 1998] M.-A. Knus, A. Merkurjev, M. Rost, and J.-P. Tignol, The book of involutions, American Mathematical Society Colloquium Publications 44, American Mathematical Society, Providence, RI, 1998. MR 2000a:16031 Zbl 0955.16001

[Lee 2014] T.-Y. Lee, "Embedding functors and their arithmetic properties", Comment. Math. Helv. 89:3 (2014), 671-717. MR 3260846 Zbl 06361417

[Prasad and Rapinchuk 2010] G. Prasad and A. S. Rapinchuk, "Local-global principles for embedding of fields with involution into simple algebras with involution", Comment. Math. Helv. 85:3 (2010), 583-645. MR 2011i:11053 Zbl 1223.11047

Received May 10, 2015. Revised September 18, 2015.

EVA BAYER-FLUCKIGER

École Polytechnique Fédérale de Lausanne

SB MATHGEOM CSAG

BATIMENT MA, STATION 8

CH-1015 LAUSANNE

SWITZERLAND

eva.bayer@epfl.ch 
TING-YU LEE

ÉCOLE Polytechnique FÉdÉRALE de LAUSANNE

SB MATHGEOM CSAG

BAtiment MA, StATION 8

CH-1015 LAUSANNE

SWITZERLAND

ting-yu.lee@epfl.ch

RAMAN PARIMALA

DEPARTMENT OF MATHEMATICS AND COMPUTER SCIENCE

EMORY UNIVERSITY

400 DOWMAN DRIVE W401

ATLANTA, GA 30322

UNITED STATES

parimala@mathcs.emory.edu 


\title{
PACIFIC JOURNAL OF MATHEMATICS
}

\author{
msp.org/pjm
}

Founded in 1951 by E. F. Beckenbach (1906-1982) and F. Wolf (1904-1989)

\section{EDITORS}

Don Blasius (Managing Editor)

Department of Mathematics

University of California

Los Angeles, CA 90095-1555

blasius@math.ucla.edu

\author{
Paul Balmer \\ Department of Mathematics \\ University of California \\ Los Angeles, CA 90095-1555 \\ balmer@math.ucla.edu \\ Robert Finn \\ Department of Mathematics \\ Stanford University \\ Stanford, CA 94305-2125 \\ finn@math.stanford.edu \\ Sorin Popa \\ Department of Mathematics \\ University of California \\ Los Angeles, CA 90095-1555 \\ popa@math.ucla.edu
}

\author{
Vyjayanthi Chari \\ Department of Mathematics \\ University of California \\ Riverside, CA 92521-0135 \\ chari@math.ucr.edu \\ Kefeng Liu \\ Department of Mathematics \\ University of California \\ Los Angeles, CA 90095-1555 \\ liu@math.ucla.edu \\ Jie Qing \\ Department of Mathematics \\ University of California \\ Santa Cruz, CA 95064 \\ qing@ cats.ucsc.edu
}

\section{PRODUCTION}

Silvio Levy, Scientific Editor, production@msp.org

\section{SUPPORTING INSTITUTIONS}

ACADEMIA SINICA, TAIPEI

CALIFORNIA INST. OF TECHNOLOGY

INST. DE MATEMÁTICA PURA E APLICADA

KEIO UNIVERSITY

MATH. SCIENCES RESEARCH INSTITUTE

NEW MEXICO STATE UNIV.

OREGON STATE UNIV.

\author{
STANFORD UNIVERSITY \\ UNIV. OF BRITISH COLUMBIA \\ UNIV. OF CALIFORNIA, BERKELEY \\ UNIV. OF CALIFORNIA, DAVIS \\ UNIV. OF CALIFORNIA, LOS ANGELES \\ UNIV. OF CALIFORNIA, RIVERSIDE \\ UNIV. OF CALIFORNIA, SAN DIEGO \\ UNIV. OF CALIF., SANTA BARBARA
}

\author{
Daryl Cooper \\ Department of Mathematics \\ University of California \\ Santa Barbara, CA 93106-3080 \\ cooper@math.ucsb.edu \\ Jiang-Hua Lu \\ Department of Mathematics \\ The University of Hong Kong \\ Pokfulam Rd., Hong Kong \\ jhlu@maths.hku.hk \\ Paul Yang \\ Department of Mathematics \\ Princeton University \\ Princeton NJ 08544-1000 \\ yang@math.princeton.edu
}

These supporting institutions contribute to the cost of publication of this Journal, but they are not owners or publishers and have no responsibility for its contents or policies.

See inside back cover or msp.org/pjm for submission instructions.

The subscription price for 2015 is US \$420/year for the electronic version, and \$570/year for print and electronic.

Subscriptions, requests for back issues and changes of subscribers address should be sent to Pacific Journal of Mathematics, P.O. Box 4163, Berkeley, CA 94704-0163, U.S.A. The Pacific Journal of Mathematics is indexed by Mathematical Reviews, Zentralblatt MATH, PASCAL CNRS Index, Referativnyi Zhurnal, Current Mathematical Publications and Web of Knowledge (Science Citation Index).

The Pacific Journal of Mathematics (ISSN 0030-8730) at the University of California, c/o Department of Mathematics, 798 Evans Hall \#3840, Berkeley, CA 94720-3840, is published twelve times a year. Periodical rate postage paid at Berkeley, CA 94704, and additional mailing offices. POSTMASTER: send address changes to Pacific Journal of Mathematics, P.O. Box 4163, Berkeley, CA 94704-0163.

PJM peer review and production are managed by EditFLOW ${ }^{\circledR}$ from Mathematical Sciences Publishers.

\section{PUBLISHED BY}

\section{mathematical sciences publishers \\ nonprofit scientific publishing}

http://msp.org/

(C) 2015 Mathematical Sciences Publishers 


\title{
PACIFIC JOURNAL OF MATHEMATICS
}

\author{
Volume 279 No. 1-2 December 2015
}

In memoriam: Robert Steinberg

Robert Steinberg (1922-2014): In memoriam V. S. VARADARAJAN

Cellularity of certain quantum endomorphism algebras

HENNING H. ANDERSEN, GUSTAV I. LEHRER and RUIBIN ZHANG

Lower bounds for essential dimensions in characteristic 2 via orthogonal representations ANTONIO BABIC and VLADIMIR CHERNOUSOV

Cocharacter-closure and spherical buildings

Michael Bate, Sebastian Herpel, Benjamin Martin and Gerhard RöHrLe

Embedding functor for classical groups and Brauer-Manin obstruction

Eva Bayer-Fluckiger, Ting-Yu LeE and Raman Parimala

On maximal tori of algebraic groups of type $G_{2}$

Constantin Beli, PhilipPe Gille and Ting-Yu LeE

On extensions of algebraic groups with finite quotient

MICHEL BRION

Essential dimension and error-correcting codes

SHANE CERNELE and ZiNOVy REICHSTEIN

Notes on the structure constants of Hecke algebras of induced representations of finite Chevalley groups

Charles W. CuRTis

Complements on disconnected reductive groups

FRANÇOIS DIGNE and JEAN MICHEL

Extending Hecke endomorphism algebras

Jie Du, Brian J. Parshall and LeOnard L. SCOTT

Products of partial normal subgroups

ELLEN HENKE

Lusztig induction and $\ell$-blocks of finite reductive groups

RADHA KESSAR and GUNTER MALLE

Free resolutions of some Schubert singularities

Manoj Kummini, Venkatramani Lakshmibai, Pramathanath Sastry and C. S. Seshadri

Free resolutions of some Schubert singularities in the Lagrangian Grassmannian

VenKatramani LAKSHMibai and ReUVEN HODGES

Distinguished unipotent elements and multiplicity-free subgroups of simple algebraic groups

Martin W. Liebeck, Gary M. Seitz and Donna M. Testerman

Action of longest element on a Hecke algebra cell module

GEORGE LUSZTIG

Generic stabilisers for actions of reductive groups

BENJAMIN MARTIN

On the equations defining affine algebraic groups

VLADIMIR L. POPOV

Smooth representations and Hecke modules in characteristic $p$

PETER SCHNEIDER

On CRDAHA and finite general linear and unitary groups

BHAMA SRINIVASAN

Weil representations of finite general linear groups and finite special linear groups PHAM HUU TIEP

The pro- $p$ Iwahori Hecke algebra of a reductive $p$-adic group, $\mathrm{V}$ (parabolic induction) MARIE-FRANCE VIGNÉRAS

Acknowledgement 